\title{
Applied methodology
}

\section{The effect of amino acid deprivation on the transfer of iron through Caco-2 cell monolayers}

Guenievre

guenievre.roussel@abdn.ac.uk

\section{Valerie Stevens}

v.stevens@abdn.ac.uk

\section{Sarah Cottin}

sarah.c.cottin@gmail.com

\section{Harry J. McArdle*}

h.mcardle@abdn.ac.uk

Rowett Institute of Nutrition and Health, University of Aberdeen, Aberdeen, UK

*Corresponding author at: Rowett Institute of Nutrition and Health, Foresterhill, Aberdeen, AB25 2ZD, UK.

\section{Abstract}

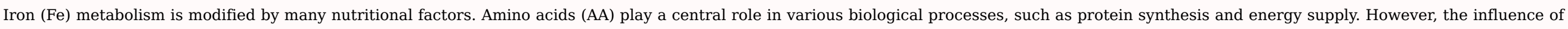

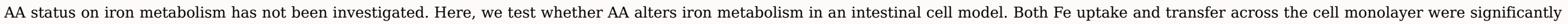

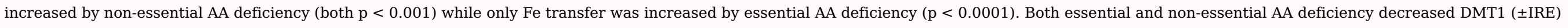

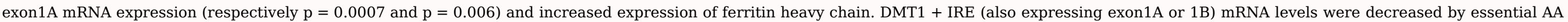

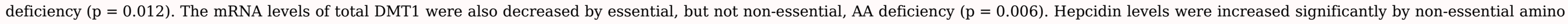

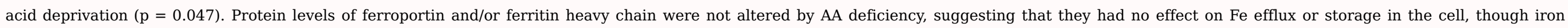

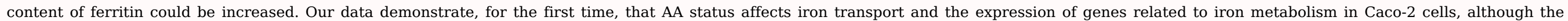

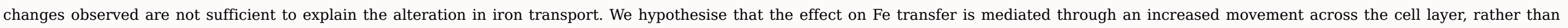
transfer across the cell membranes.

Keywords: Amino acid deprivation; Nutrient transfer; Gut transport

\section{Introduction}

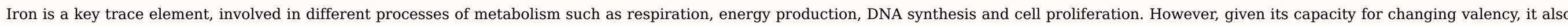
has potential for toxicity at excessive levels. Consequently, a tight regulation of absorption and excretion has evolved.

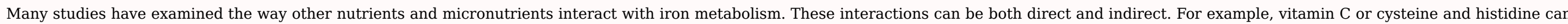

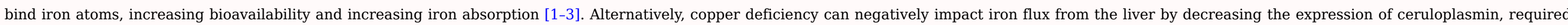

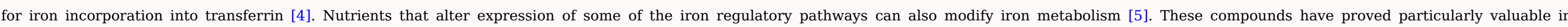
elucidating the regulation of iron.

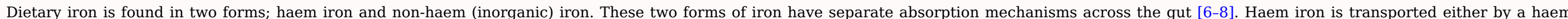




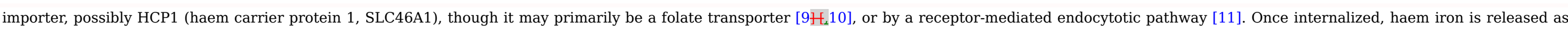

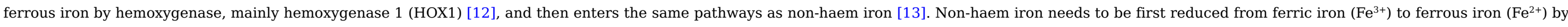

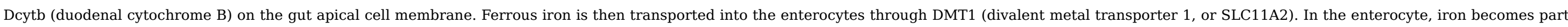

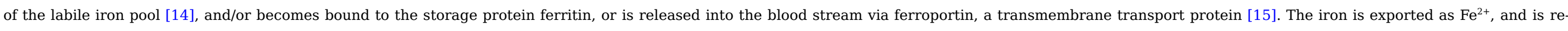
oxidised by hephaestin prior to incorporation into transferrin.

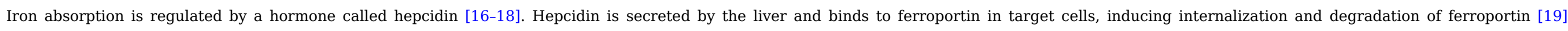
Hepcidin is not only regulated by iron bioavailability and hepatic iron storage but also by other factors including erythropoietic activity, hypoxia and inflammatory or infectious disease.

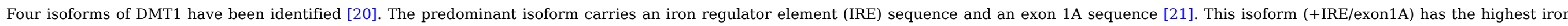

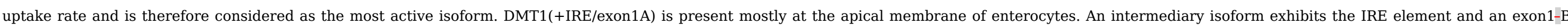

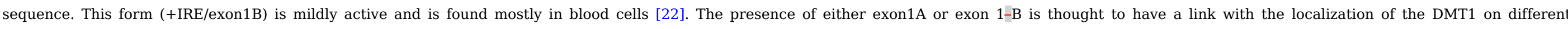

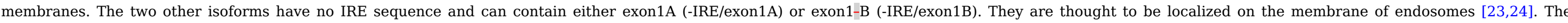

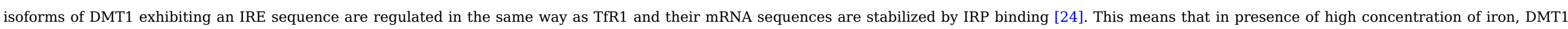
mRNA is degraded, while in iron deficiency, translation of DMT1 mRNA translation is increased.

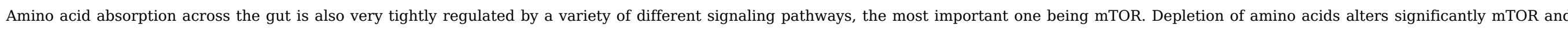

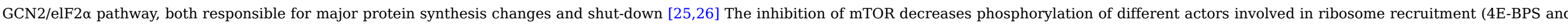

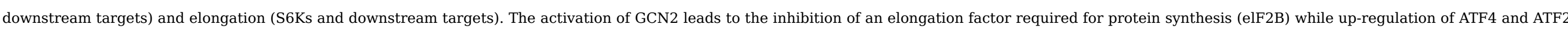

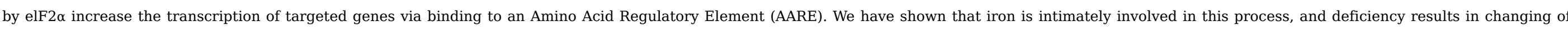

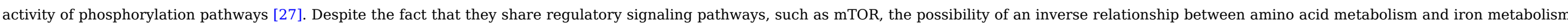

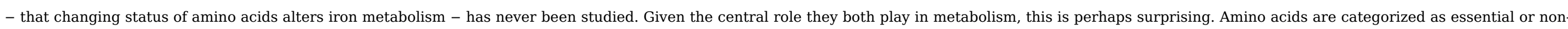
essential according to their ability to be synthesized by human cells. We tested the hypothesis that altering their levels will, in turn, affect iron metabolism in gut cells.

\section{Material and methods}

\subsection{Caco-2 cell line}

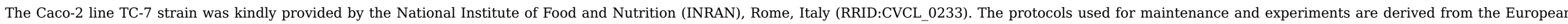

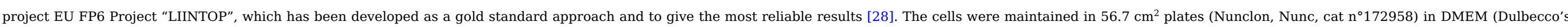

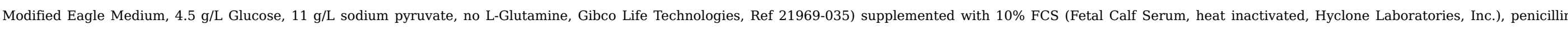

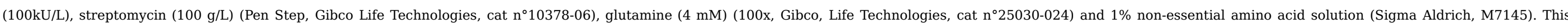
DMEM preparation is referred as 'complete DMEM' below.

\subsection{Passaging and viability}

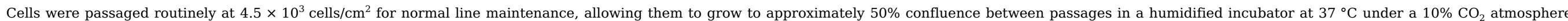
[28,29]. Cells were used for experiments between passage 10 and passage 33 as recommended [30,31].

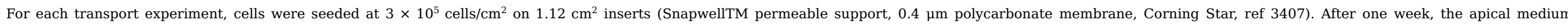

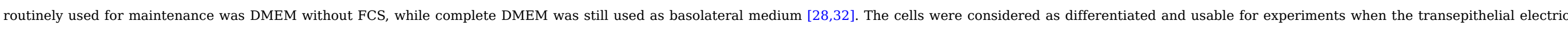
resistance (TEER, $\Omega \cdot \mathrm{em} 2$ ) reached $\left.240 \Omega . \mathrm{cm}^{2}\right)$ reached $240 \Omega \mathrm{cm}^{2}$, which takes about 21 days [33].

\subsection{Amino acid depleted media}




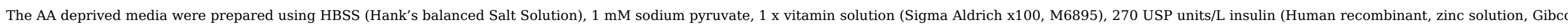

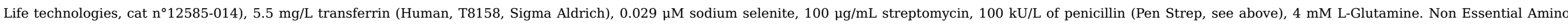

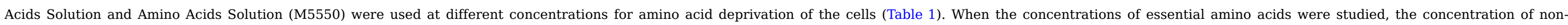
essential amino acids was kept constant at $100 \%$ and vice versa. All media were adjusted to a pH of 7.4.

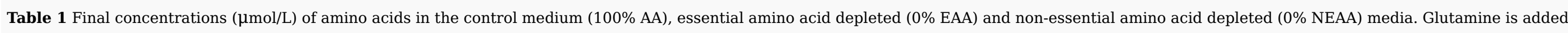
separately to control and essential amino acid depleted media, and is considered as a non-essential amino acid.

alt-text: Table 1

$100 \% \mathrm{AA}(\mu \mathrm{M})$

$0 \% \operatorname{EAA}(\mu \mathrm{M})$

$0 \% \operatorname{NEAA}(\mu \mathrm{M})$

ESS and COND ESS (M5550)

$\pm \underline{\perp-A r g i n i n e}$

ECYSTEINE-Cysteine $2 \mathrm{HCl}$

-

EL-Isoleucine

$\pm \mathrm{L}$-Leucine

EEYSTE-Lysine $\cdot \mathrm{HCl}$

$\pm \underline{\underline{L}-}-$ Methionine

EL-Phenylalanine

$\underline{ \pm L}$-Threonine

ETPYPTOPHAN1020.010.2+L-Tryptophan

\section{L-Tyrosine}

$\pm \underline{E}-$ Valine

126.4

31.3

42.0

52.5

52.4

72.5

15.1

33.0

47.6

$\underline{10.2}$

36.0

46.8

NON ESS (M7145)

fL-Alanine

LAsparagine:H2O

$\pm \underline{\mathrm{L}}-$ Aspartic Acid

fL-Glutamic Acid

Glycine

世L-Proline

$\pm \underline{\mathrm{E}}-$ Serine

$\pm \underline{\text { L-Glutamine }}$

\section{$2.4{ }^{59} \mathrm{Fe}$ transepithelial transport}

8.9

13.3

14.7

7.5

11.5

10.5

584.6

\begin{tabular}{|l|l|}
\hline .0 & 126.4 \\
\hline 0.0 & 31.3 \\
\hline 0.0 & 42.0 \\
\hline 0.0 & 52.5 \\
\hline 0.0 & 52.4 \\
\hline 0.0 & 72.5 \\
\hline 0.0 & 15.1 \\
\hline 0.0 & 33.0 \\
\hline 0.0 & 47.6 \\
\hline 0.0 & 10.2 \\
\hline 0.0 & 36.0 \\
\hline
\end{tabular}

8.9

15.0

13.3

14.7

7.5

11.5

10.5

584.6
0.0

0.0

0.0

0.0

0.0

0.0

0.0

0.0 


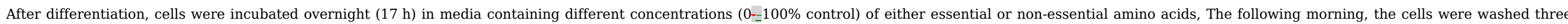
times in PBS (Phosphate Buffered Saline) prior to addition of the experimental media.

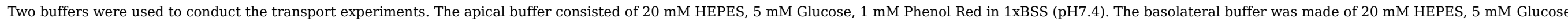

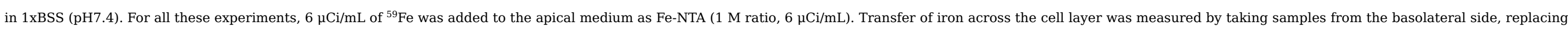
the sample volume with fresh buffer, every 30 see.

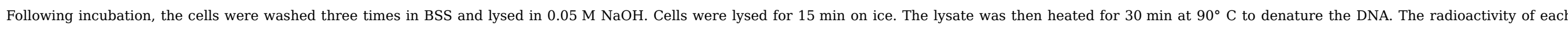
sample (basolateral media and cell lysates) was measured in a gamma counter (Wizard 3" 1480, Automatic gamma counter, Perkin Elmer) calibrated against standards of the incubation medium.

\subsection{RT-qPCR}

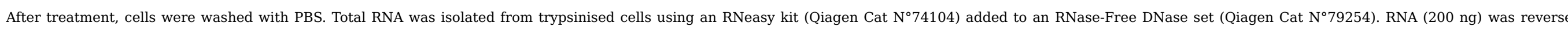

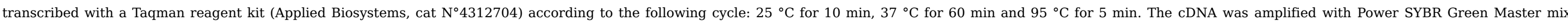

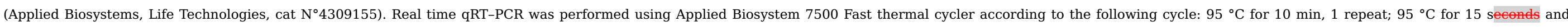

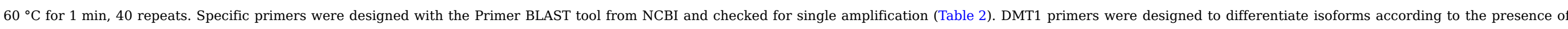
IRE (DMT1 +IRE (exon1A or 1B)), the absence of IRE (DMT1 -IRE (exon 1A or 1B)), the presence of exon 1A (DMT1 ( \pm IRE) exon1A), or the presence of exon 1-B (DMT1 ( \pm IRE) exon1B).

Table 2 Nucleotide sequence of primers used to determine levels of the genes measured in this paper.

alt-text: Table 2

\begin{tabular}{|c|c|c|}
\hline Gene of interest & Type of sequence & Nucleotide sequence \\
\hline \multirow[t]{2}{*}{ DMT1(+IRE) } & Forward & ACC TTA GAA CTG GAT TAG GGT \\
\hline & Reverse & AAG GAT AAA CTG AGC TCG CCC \\
\hline \multirow[t]{2}{*}{ DMT1(-IRE) } & Forward & AAA TGT AGG TCA GAG ACC CAC \\
\hline & Reverse & AAT CCC AGA GTC CAA GAC ACA \\
\hline \multirow[t]{2}{*}{ DMT1 (exon1A) } & Forward & AGG CAG CTC CAC ACT GTG AAC \\
\hline & Reverse & ACA TCT TCT GTT CAG GAC CCA \\
\hline \multirow[t]{2}{*}{ DMT1(exon1b) } & Forward & TGG CGC TGG CTC CCG GAA TAT \\
\hline & Reverse & GAA TAT GAT TCT TAC CAG CTC \\
\hline \multirow[t]{2}{*}{$18 \mathrm{~S}$} & Forward & AGA AAC GGC TAC CAC ATC CCA \\
\hline & Reverse & CAC CAG ACT TGC СCT CCA \\
\hline
\end{tabular}

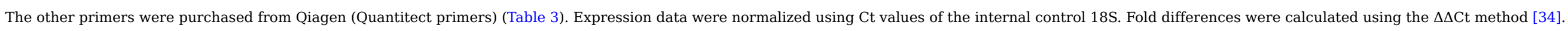

Table 3 Product number and company of purchase of commercially available primers. 


\begin{tabular}{|l|l|l|l}
\hline \multicolumn{1}{|c|}{ Gene of interest } & \multicolumn{2}{c}{ Company of purchase } \\
\hline Ferritin Light (Hs_FTL_1_SG) & Qiagen (Quantitect primers) & QT00055860 \\
\hline Ferritin Heavy (Hs_FTH1_1_SG) & Qiagen (Quantitect primers) & QT00072681 \\
\hline Ferroportin (Hs_SLC40A1_1_SG) & Qiagen (Quantitect primers) & QT00094843 \\
\hline Hepcidin (Hs_HAMP_1_SG) & Qiagen (Quantitect primers) & QT00200522 \\
\hline Hephaestin (Hs_HEPH_1_SG) & Qiagen (Quantitect primers) \\
\hline
\end{tabular}

\subsection{Protein expression of ferroportin and intracellular and extracellular ferritin heavy chain by ELISA}

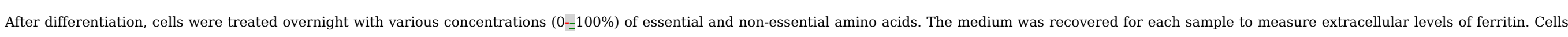

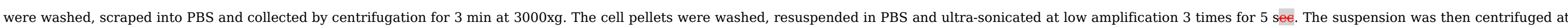

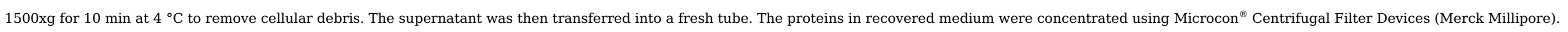

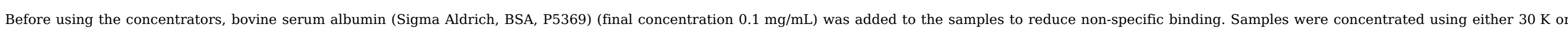

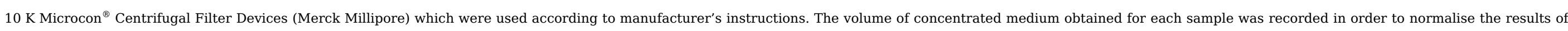
the ELISA.

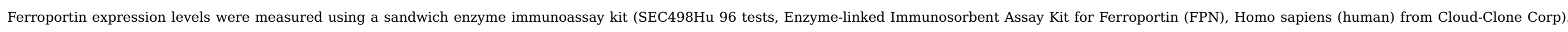

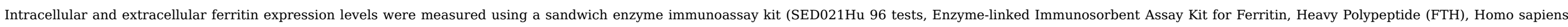
(human) from Cloud-Clone Corp).

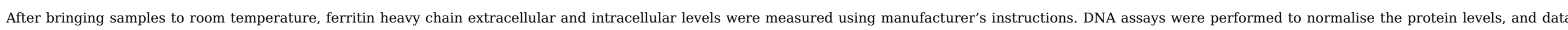
were then expressed as percentage of the control (100\% amino acids).

\subsection{Statistical analysis}

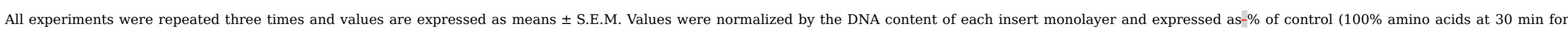
transfer experiments, $100 \%$ amino acids for RT-qPCR and protein expression).

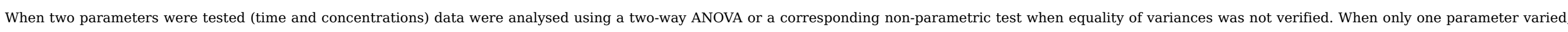
statistical significance was determined by one-way ANOVA or corresponding non-parametric test when equality of variance was not verified.

All tests were performed using Graph-Pad Prism 5 statistical software (http://www.graphpad.com/, RRID:SCR_002798) and $\mathbf{p} \leq 0.05$ was considered as significant.

\section{Results}

\section{1 ${ }^{59}$ Fe transepithelial transport is increased by both essential and non-essential amino acid deprivation}

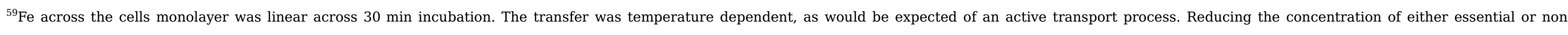

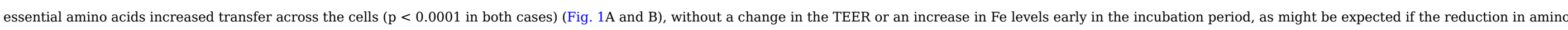

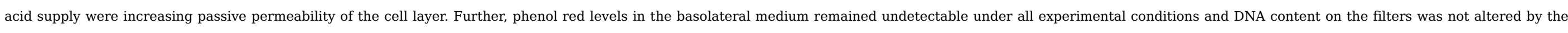
treatments (Appendix A, Fig. A1). 

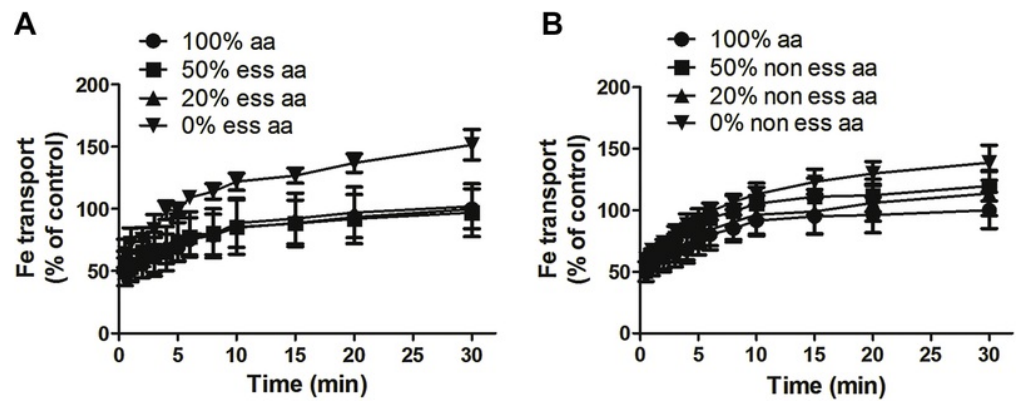

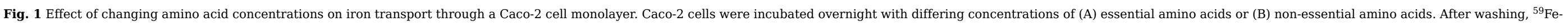

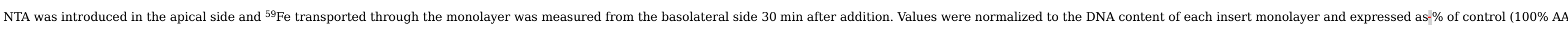

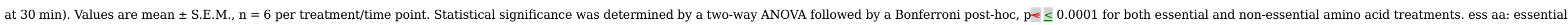
amino acids, non ess aa: non-essential amino acids, aa: amino acids.

\section{alt-text: Fig. 1}

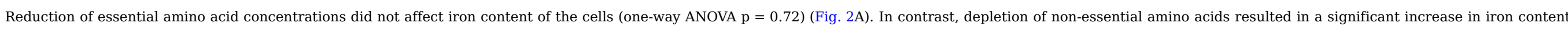
$(p<0.0001)$ (Fig. 2B)
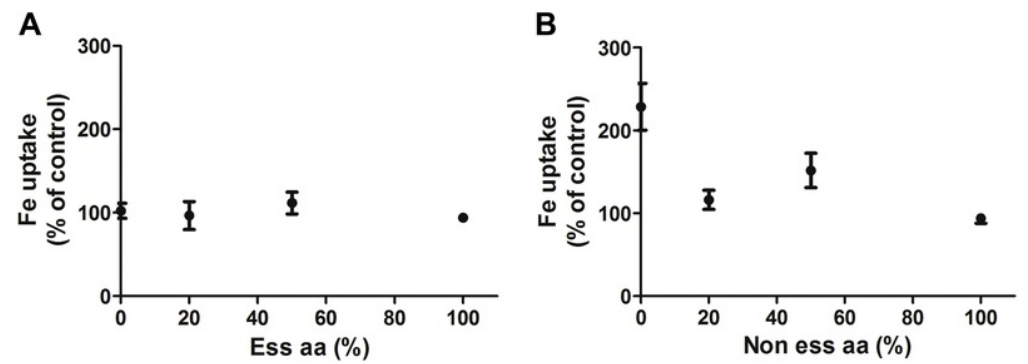

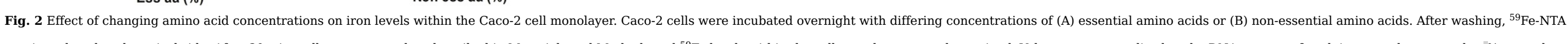

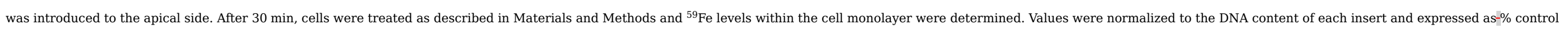

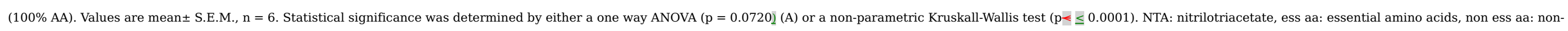
essential amino acids.

\section{alt-text: Fig. 2}

\subsection{Gene expression}

The expression of different genes involved in iron metabolism was measured following essential or non-essential amino acid depletion.

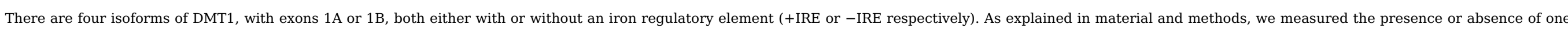

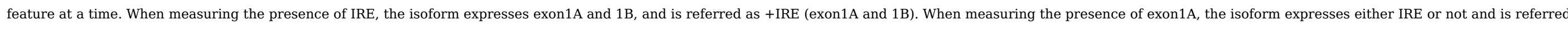

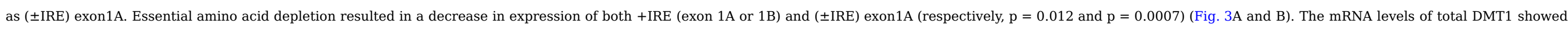
an overall significant decrease in expression $(p=0.006$ ) (Fig. 3-C). In contrast to DMT1, ferritin heavy chain mRNA levels significantly increased with essential amino acid depletion ( $p=0.01$ ) (Fig. 3D). 


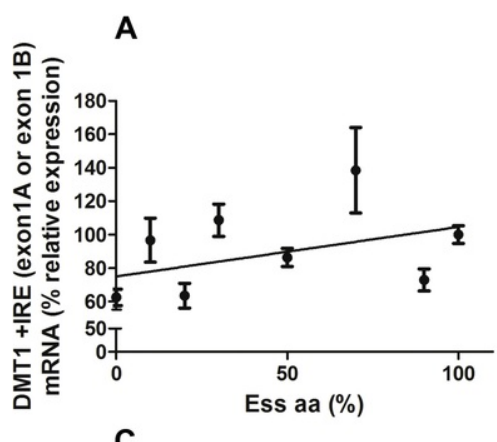

C

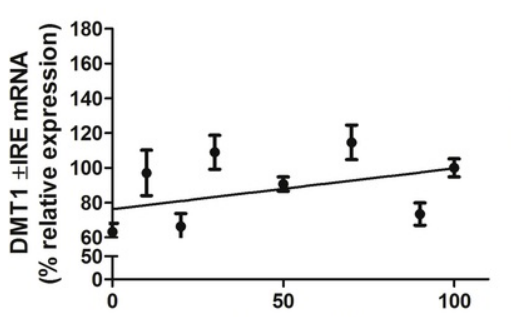

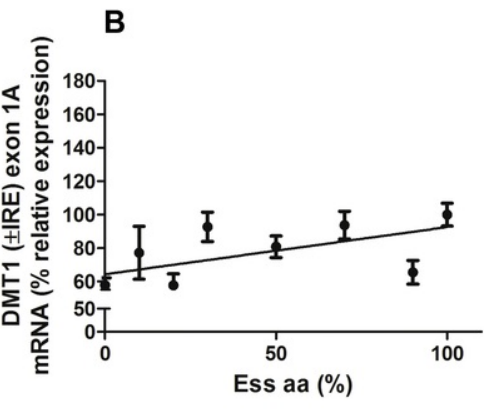

D

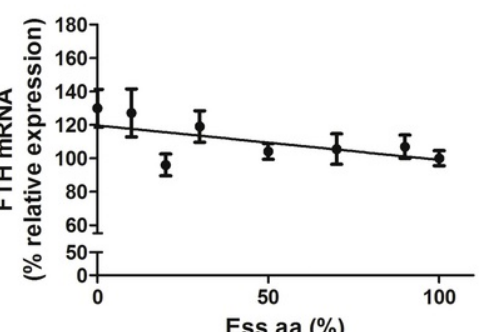

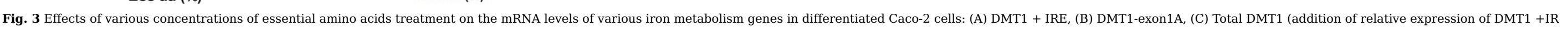

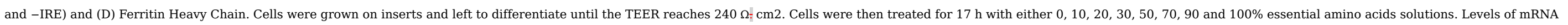

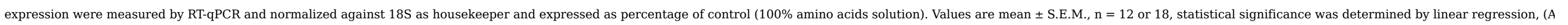

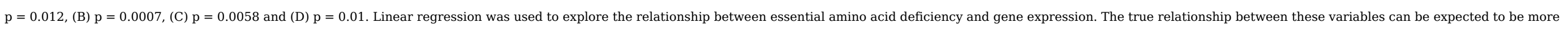

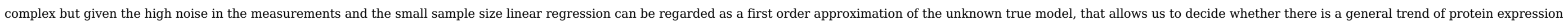

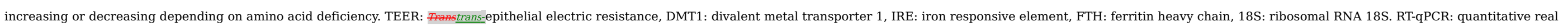
time polymerase chain reaction, ess aa: essential amino acids.

\section{alt-text: Fig. 3}

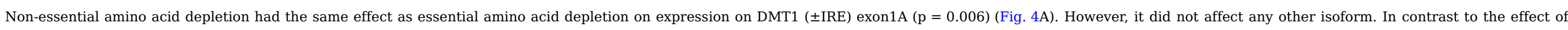

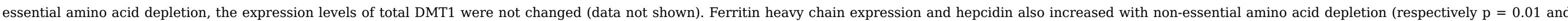
$\mathrm{p}=0.047$ ) (Fig. $4 \mathrm{~B}$ and $\mathrm{C}$ ). 


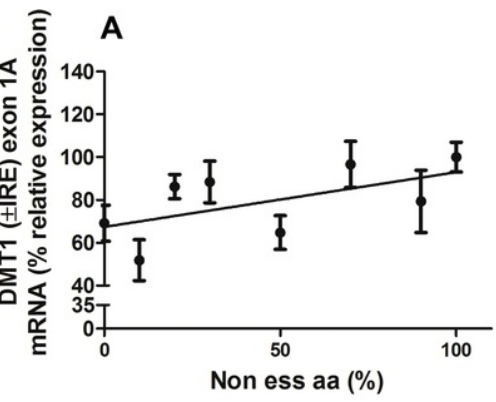

B

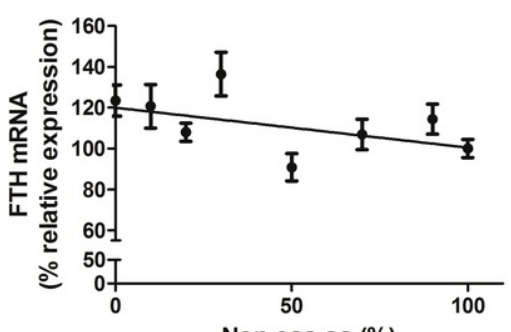

Non ess aa (\%)

C

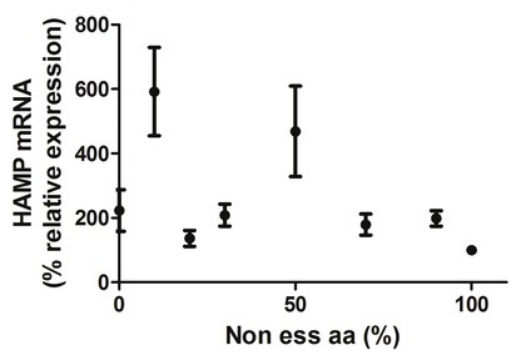

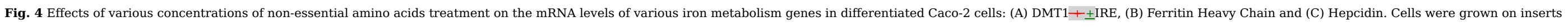

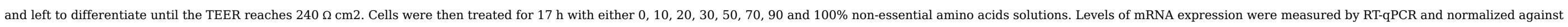

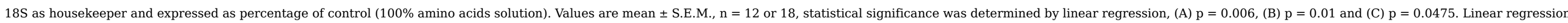

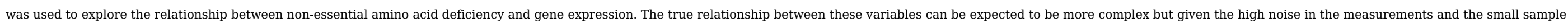

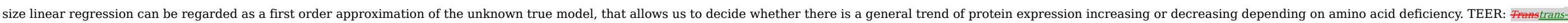

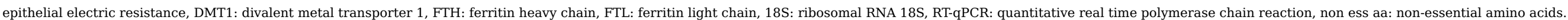
alt-text: Fig. 4

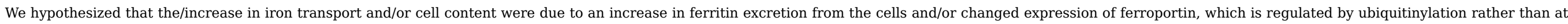
mRNA level [12]. However, protein levels of ferritin and of ferroportin were not significantly altered by any of the treatments (Appendix A Fig. A2 and Fig. A3).

\section{Discussion}

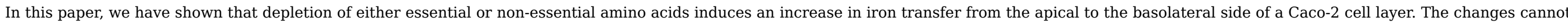

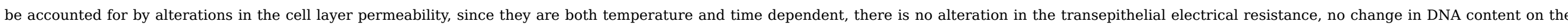




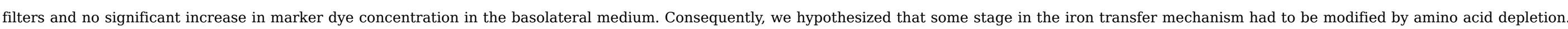

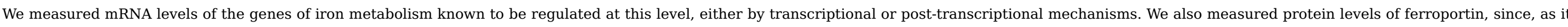
is regulated by ubiquitinylation, mRNA is not necessarily an accurate reflection of activity

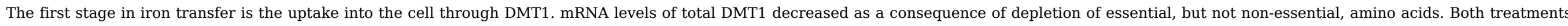

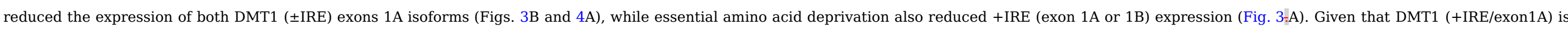

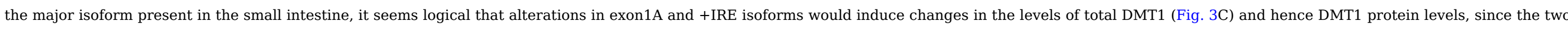

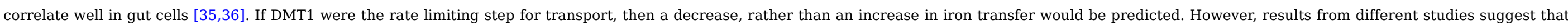

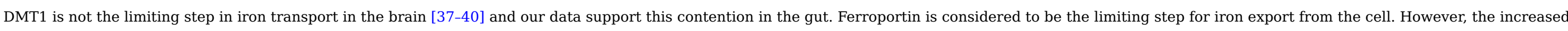
transport of iron in both types of AA depletion cannot be explained by changes in ferroportin expression, which remained constant in our study.

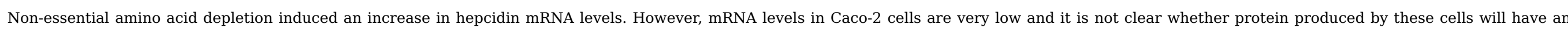

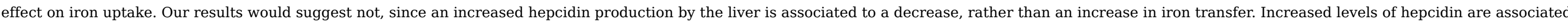

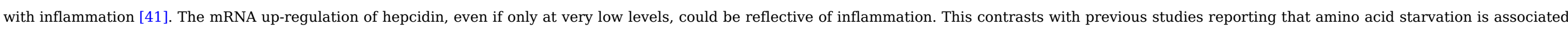

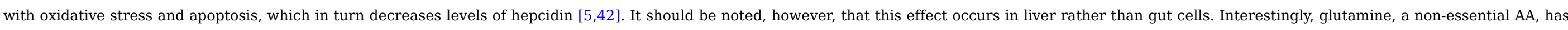

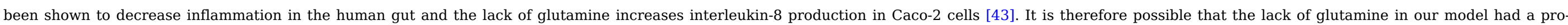

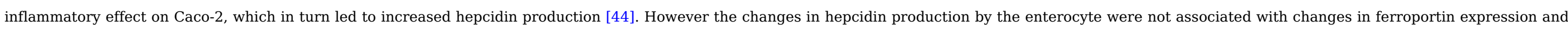
its biological role remains to be established.

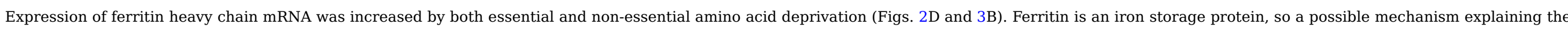

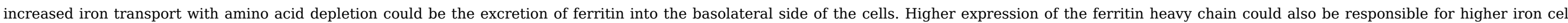

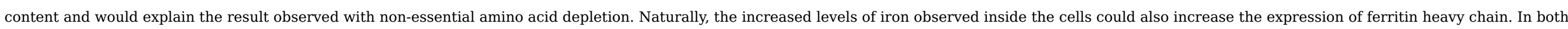

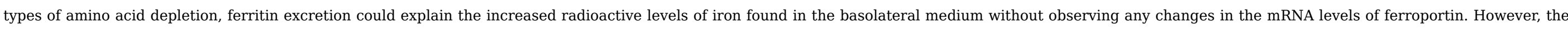

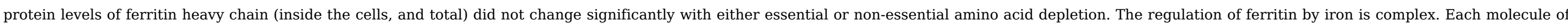

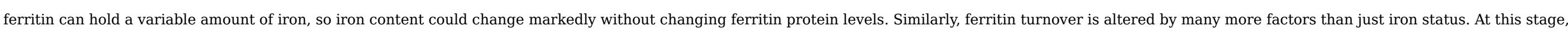

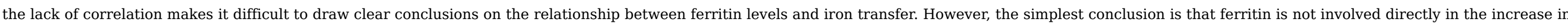
transfer.

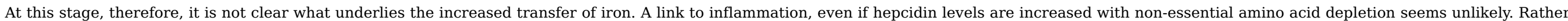

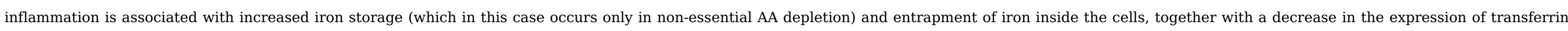
and the internalization and degradation of ferroportin, caused by hepcidin up-regulation.

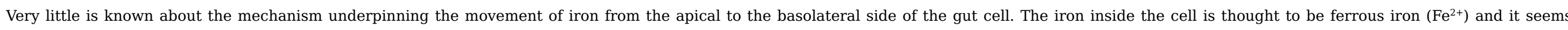

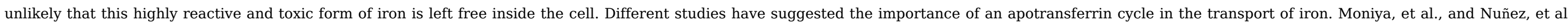

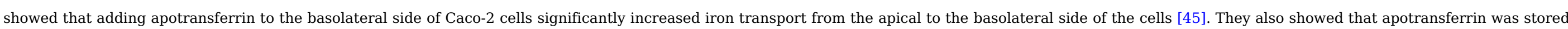

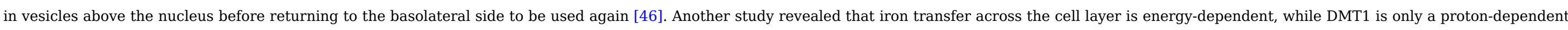

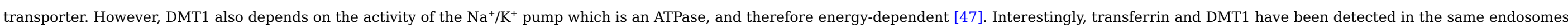

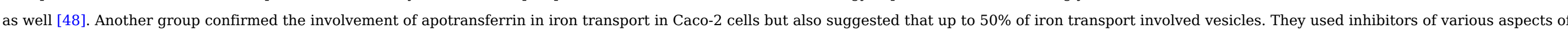

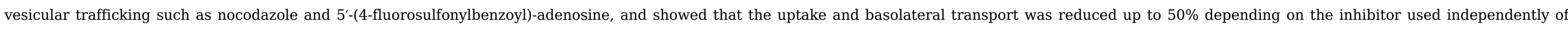

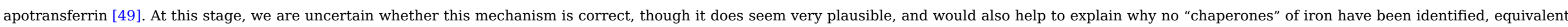
to those clearly identified for copper [50,51]. 


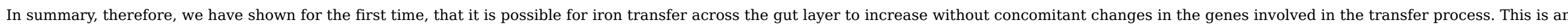
intriguing outcome, and one that deserves further investigation.

\section{Funding Source}

Rural and Environmental Scientific and Analytical Services, the Scottish Government

\section{Acknowledgments}

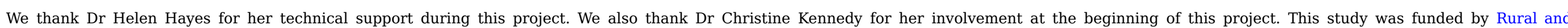
Environmental Scientific and Advisory Service of Scottish Government.

\section{Appendix A}

A
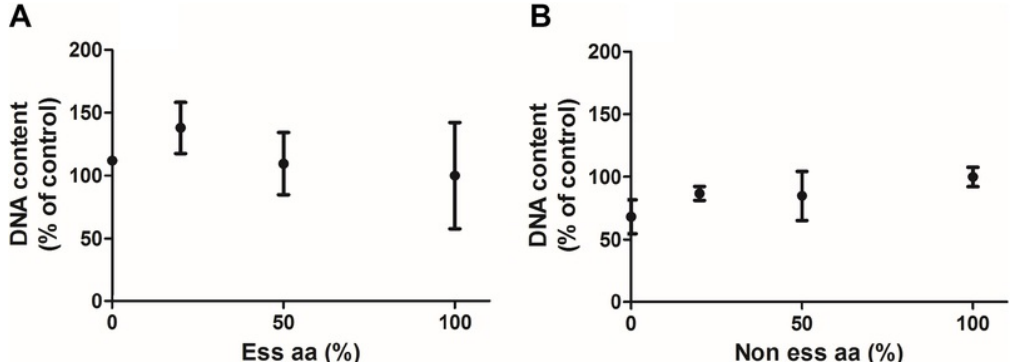

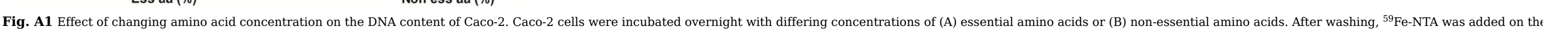

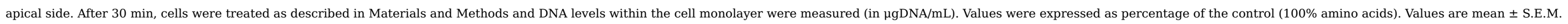
$\mathrm{n}=3$, statistical significance was determined by a one-way ANOVA, $\mathrm{p}=0.43$ (EAA) and $\mathrm{p}=0.77$ (NEAA). Ess aa: essential amino acids, non ess aa: non-essential amino acids, aa: amino acids, DNA: deoxyribonucleic acid. alt-text: Fig. A1

A

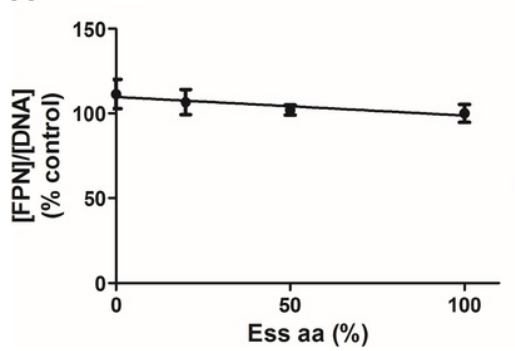

B

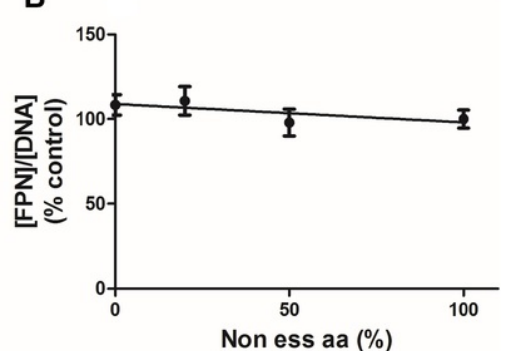

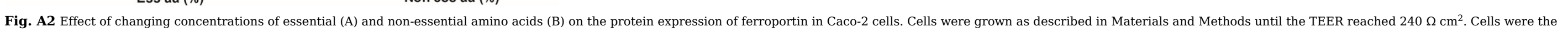

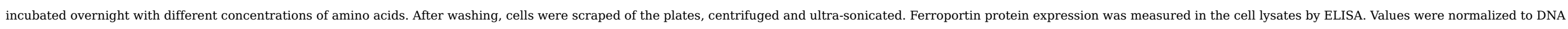

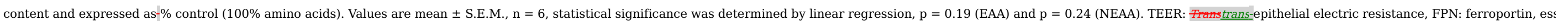
aa: essential amino acids, non ess aa: non-essential amino acids. 

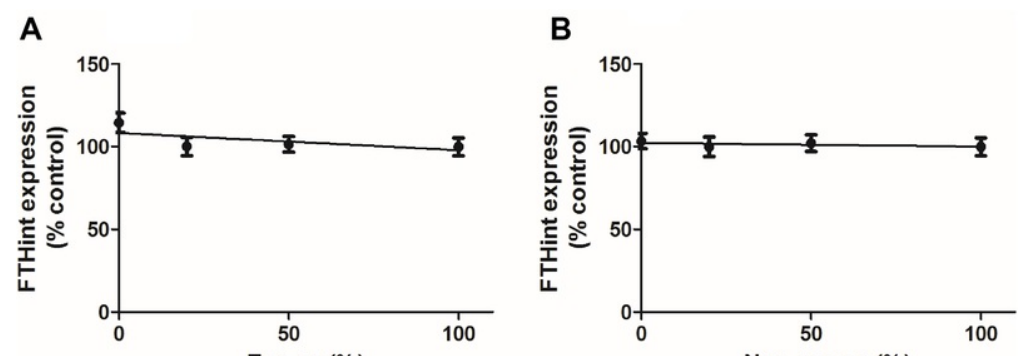

Ess aa $(\%$

Non ess aa (\%)

C

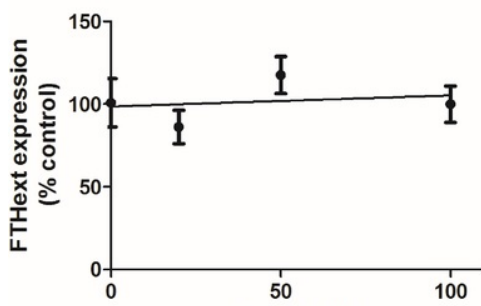

Ess aa (\%)

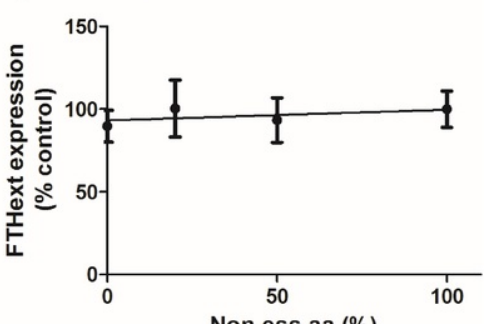

E

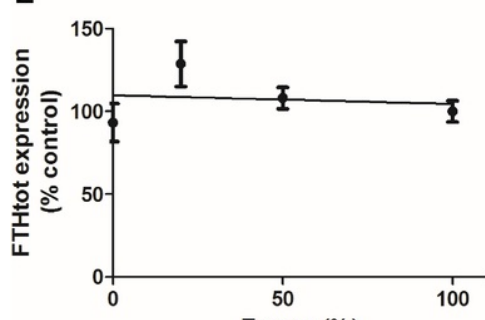

Ess aa (\%)

100

F

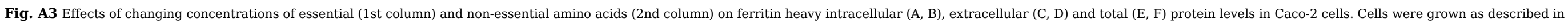

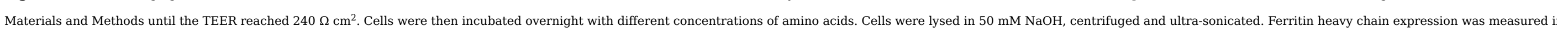

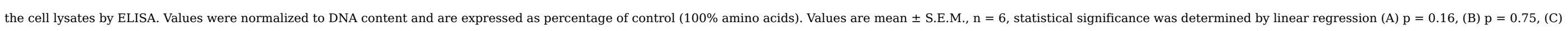

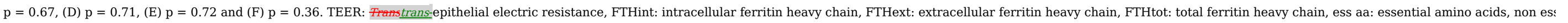
aa: non-essential amino acids.

\section{alt-text: Fig. A3}

\section{References}

[1] R.P. Glahn and D.R. Van Campen, Iron uptake is enhanced in Caco-2 cell monolayers by cysteine and reduced cysteinyl glycine, Fhe foumal of NutritionI. Nutr. 127, $1997,642-647$.

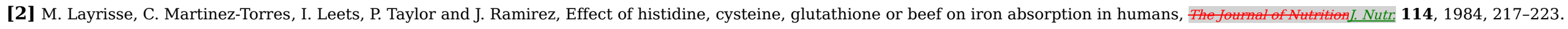

[3] V. Iyengar, R. Pullakhandam and K.M. Nair, Dietary ligands as determinants of iron-zinc interactions at the absorptive enterocyte, JFood Set. Food Sci. 75, 2010, H260-4.

[4] D.M. de Silva and S.D. Aust, Ferritin and ceruloplasmin in oxidative damage: review and recent findings, CanJ Physiol Pharmacos. I. Physiol. Pharmacol. 71, 1993, 715-720.

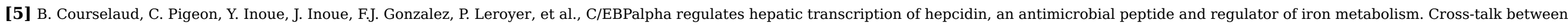


C/EBP pathway and iron metabolism, The Joumal of Biological Chemistry. Biol. Chem. 277, 2002, 41163-41170.

[6] L.L. Dunn, Y.S. Rahmanto and D.R. Richardson, Iron uptake and metabolism in the new millennium, Trends Gell BiologyCell Biol. 17, 2007, 93-100.

[7] J. Umbreit, Iron deficiency: a concise review, Am JHemato. J. Hematol. 78, 2005, 225-231.

[8] M.B. Zimmermann and R.F. Hurrell, Nutritional iron deficiency, Lancet 370, 2007, 511-520.

[9] M. Shayeghi, G.O. Latunde-Dada, J.S. Oakhill, A.H. Laftah, K. Takeuchi, N. Halliday, et al., Identification of an intestinal heme transporter, Cell 122, 2005 , 789-801.

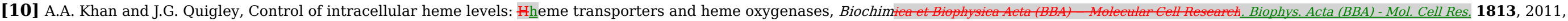
668-682.

[11] A.R. West and P.S. Oates, Mechanisms of heme iron absorption: Eçurrent questions and controversies, World Joumal of Gastroenterology. Gastroenterol. 14, 2008, 4101-4110.

[12] C.D. Ferris, S.R. Jaffrey, A. Sawa, M. Takahashi, S.D. Brady, R.K. Barrow, et al., Haem oxygenase-1 prevents cell death by regulating cellular iron, Nat-Cell Bite. Cell Biol. 1, 1999, 152-157.

[13] M. Munoz, I. Villar and J.A. Garcia-Erce, An update on iron physiology, World jastroenterol. Gastroenterol. 15, 2009, 4617-4626.

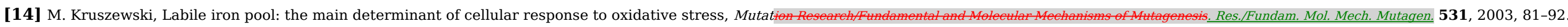

[15] O. Han and E.Y. Kim, Colocalization of ferroportin-1 with hephaestin on the basolateral membrane of human intestinal absorptive cells, J-Cell Biochem, Cell. Biochem. 101, 2007, 1000-1010.

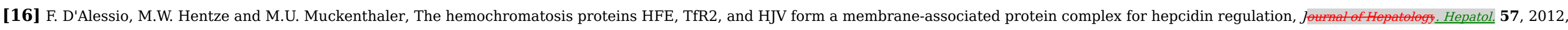
$1052-1060$.

[17] R. Evstatiev and C. Gasche, Iron sensing and signalling, Gut 61, 2011, 933-952.

[18] M.W. Hentze, M.U. Muckenthaler, B. Galy and C. Camaschella, Two to Tango: Regulation of Mammalian Iron Mregulation of mammalian iron metabolism, Cell 142, $2010,24-38$.

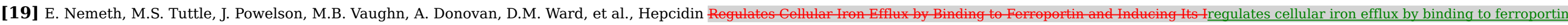
and inducing its internalization, Science 306, 2004, 2090-2093.

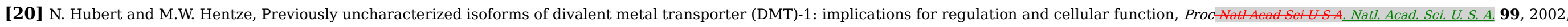
12345-12350.

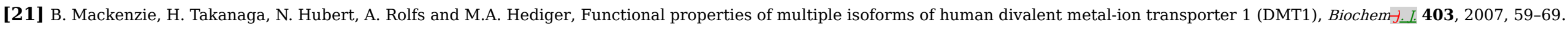

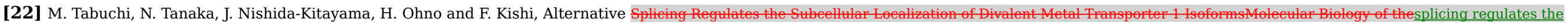
subcellular localization of divalent metal transporter 1 isoforms, Mol. Biol. Cell 13, 2002, 4371-4387.

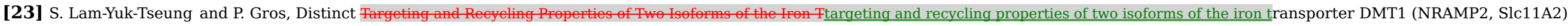
Biochemistry 45, 2006, 2294-2301.

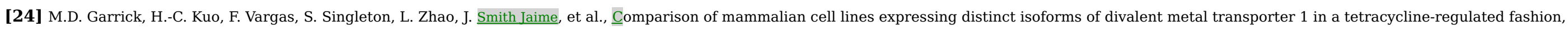
Biochemieal foumat. 398, 2006, 539-546.

[25] C.G. Proud, Control of the translational machinery by amino acids, The American journal of elinieal nutritionAm. J. Clin. Nutr. 99, 2014, 231S-236S.

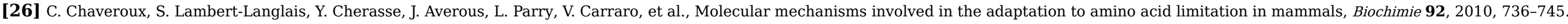




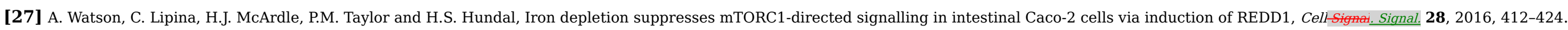

[28] S. Ferruzza, C. Rossi, M.L. Scarino and Y. Sambuy, A protocol for differentiation of human intestinal Caco-2 cells in asymmetric serum-containing medium, Toxicol_ In Vitro 2012.

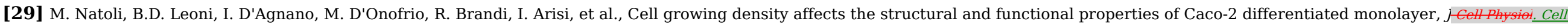
Physiol. 226, 2011, 1531-1543.

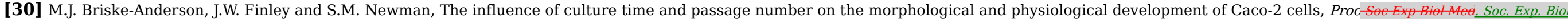
Med. 214, 1997, 248-257.

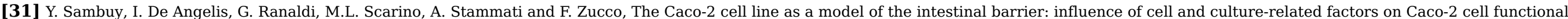
characteristics, Cell Biorogy and Toxieology. Toxicol. 21, 2005, 1-26.

[32] S. Ferruzza, C. Rossi, Y. Sambuy and M.L. Scarino, Serum-reduced and serum-free media for differentiation of Caco-2 cells, ALTEX 30, 2013, 159-168.

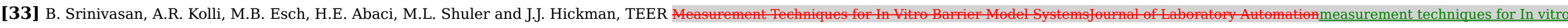
barrier model systems, L. Lab. Autom. 20, 2015, 107-126.

[34] K.J. Livak and T.D. Schmittgen, Analysis of relative gene expression data using real-time quantitative PCR and the 2(-Delta Delta C(T)) Method, NMethods 25, 2001, 402-408.

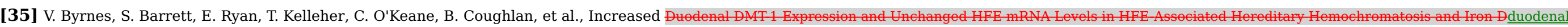
DMT-1 expression and unchanged HFE mRNA levels in HFE-associated hereditary hemochromatosis and iron deficiency, Blood CellsMolecules, and Diseases. Mol. Dis. 29, 2002, 251-260.

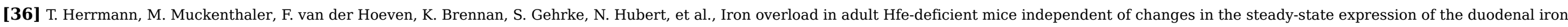
transporters DMT1 and Ireg1/ferroportin, Journal of Molectlar Medicine. Mol. Med. 82, 2004, 39-48.

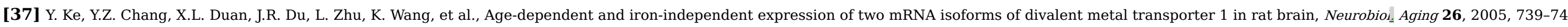

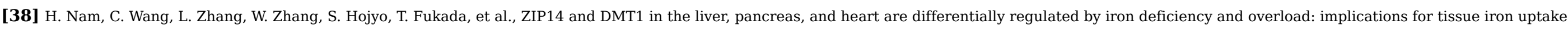
in iron-related disorders, Haematologica 2013, epub.

[39] B. Mackenzie and J.D. Erickson, Sodium-coupled neutral amino acid (System N/A) transporters of the SLC38 gene family, Pflugers Arch 447, $2004,784-795$.

[40] D.M. Frazer and G.J. Anderson, The orchestration of body iron intake: how and where do enterocytes receive their cues? Blood Cells MoiDis. Dis. 30, $2003,288-297$.

[41] A. Kali, M.V.P. Charles and R.S.K. Seetharam, Hepcidin - Aa novel biomarker with changing trends, Pharmacognesy Reviews. Rev 9, 2015, 35-40.

[42] D. Darshan, D.M. Frazer and G.J. Anderson, Molecular basis of iron-loading disorders, Expert Rev Mol Mea. Mol. Med. 12, 2010 , e36.

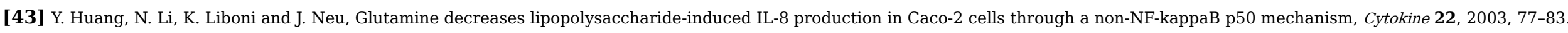

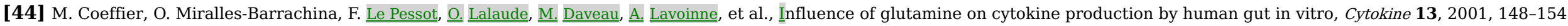

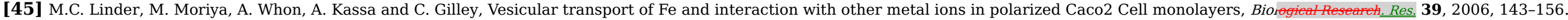

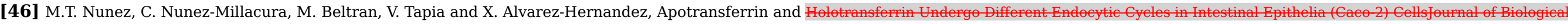
Chemistryholotransferrin undergo different endocytic cycles in intestinal epithelia (Caco-2) cells, J. Biol. Chem. 272, 1997, 19425-19428.

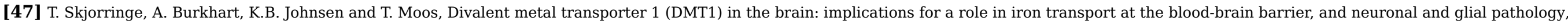
Front Mol Neuresei. Mol. Neurosci. 8, 2015, 19. 


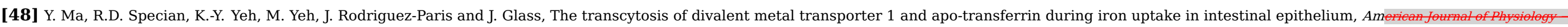
Gastrointestimal and Liver Physiology. J. Physiol. Gastrointest. Liver Physiol. 283, 2002, G965-G974.

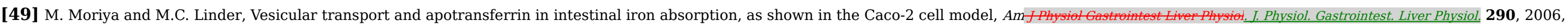
G301-9.

[50] M.D. Harrison, C.E. Jones and C.T. Dameron, Copper chaperones: function, structure and copper-binding properties, JBiol frorg Chem. Biol. Inorg. Chem. 4, 1999, 145-153.

[51] L.M. Gaetke, H.S. Chow-Johnson and C.K. Chow, Copper: toxicological relevance and mechanisms, Arch-Toxicol.Toxicol. 88, 2014, $1929-1938$.

\section{Queries and Answers}

Query: Please check the document header that has been updated, and correct if necessary.

Answer: Header is OK

Query: The author names have been tagged as given names and surnames (surnames are highlighted in teal color). Please confirm if they have been identified correctly.

Answer: These are correct

Query: Please check the hierarchy of the section headings.

Answer: Hierarchy is OK

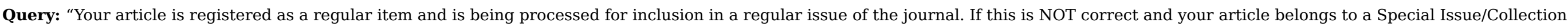
please contact p.kamaraj@elsevier.com immediately prior to returning your corrections."

Answer: This is correct

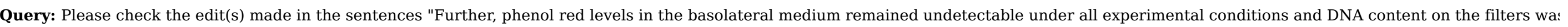

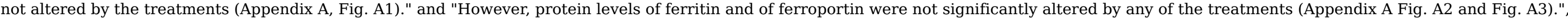
and correct if necessary.

Answer: These are OK

Query: Please check the presentation of Appendix A, and correct if necessary.

Answer: Appendix A is OK

Query: Please check the presentation of Funding, and correct if necessary.

Answer: OK

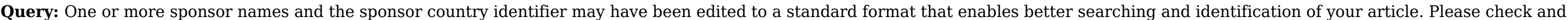
correct if necessary.

Answer: OK

Query: Please check the presentation of Ref. [14], and correct if necessary.

Answer: OK

Query: Please provide the volume number and page range for Refs. [28] and [38].

Answer: Ref 22 Vol:26 pp 1252-1255. Ref 38: Vol 98 pp 1049-1057 
Query: Please check the presentation of all the figure captions, and correct if necessary.

Answer: OK

Query: Please check the presentation of all the tables, and correct if necessary

Answer: OK 